(C) Tick paralysis

(D) Myasthenia gravis

3. If a patient has developed aspiration pneumonia, what is the most appropriate method of feeding?

(A) Balls of soft food fed from a height with the patient remaining raised after finishing
(B) Soft food from a bowl on the floor then raising the patient once they have finished eating

(C) Dry food fed from a height, patient is not kept raised

(D) Dry food from a bowl on the floor and patient is never raised

\section{What nurses need to know about inflammatory brain disease}

\section{Raquel Trevail}

MUA includes a variety of neurological disorders in both dogs and cats. It generally involves more than one part of the central nervous system and the presenting neurological signs can localize to a specific area of the brain or present with multifocal signs. Inflammatory brain diseases can also be further divided into infectious and non-infectious, with non-infectious aetiologies being more common than infectious aetiologies.

MUA is secondary to an immune-mediated response where the body 'fights' itself. No specific trigger has been identified and a genetic background is found in some breeds. The presenting clinical signs vary depending on which areas are affected and can vary from solely lethargy, to seizures, multifocal neurological signs to coma. The spinal cord can be affected in some cases and, in these, subsequent neurological signs such as pain or changes in gait can be seen.

The diagnosis of inflammatory non-infectious disease is generally achieved with advanced imaging, preferably magnetic resonance imaging (MRI) due to its increased sensitivity, and cerebrospinal fluid (CSF) analysis when safe to perform. The latter is only recommended following advanced imaging as most cases are associated with changes in intracranial pressure. Serology for infectious diseases and polymerase chain reaction on the CSF can be considered to investigate infectious aetiologies.

Treatment depends on the initial complaint, cause and severity of neurological deficits. In non-infectious MUA, immunosuppressive therapy is required. Monotherapy or combination therapy can be chosen. There is no proven superior treatment or combination of treatments and therefore the choice is made on experience with certain drugs, severity of presentation, the need for monitoring and associated costs. The aim is for the patient to return to normal and to achieve control with the lowest dose of medication possible. It is rare for patients to be cured, and therefore long-term therapy is the realistic expectation. It is possible to discontinue medication in some cases without relapses but, on the other hand, some cases will deteriorate despite aggressive therapy. It is not possible to predict the response to treatment and therefore trial treatment is generally recommended. Without treatment, the prognosis is poor and survival times are short.

While in hospital the care required depends on the severity of the clinical signs. It is important to remember that the severity of the signs may or may not be related to prognosis. Certain presentations, such as severe changes in mental status and lack of gag reflex, are more likely to be associated with complications such as aspiration pneumonia that can also compromise recovery. Close monitoring in these cases is paramount to establish response to treatment, and correct information at time of discharge is essential for a successful management.

\section{KEY LEARNING OBJECTIVES}

- Meningoencephalitis of unknown aetiology (MUA) refers to a variety of neurological disorders and therefore the clinical presentation can vary

- Recognize that there is a breed predisposition to each type of MUA but any breed can be affected

- Understand that treatment and prognosis vary depending on the underlying aetiology but a definitive diagnosis can only be achieved by brain biopsy or postmortem examination

\section{MULTIPLE CHOICE QUESTIONS}

1. Which breed is necrotizing leukoencephalitis more likely to be seen in?
(A) Pugs
(C) Yorkshire Terriers
(B) Chihuahuas
(D) Jack Russell Terriers

2. What is the best test/tests to perform to reach a diagnosis of MUA?

(A) MRI only

(B) CSF only

(C) DNA test

(D) MRI, CSF analysis and serology

3. A 2-year-old, neutered male Pug presented with seizures. A diagnosis of MUA was reached by means of MRI and CSF analysis. Cytarabine was started in hospital. How long should the owners be careful with urine and faeces?
(A) 1 month
(C) 2 weeks
(B) 3 days
(D) 1 week 